Case Report

\title{
Severe COVID-19 in Third Trimester Pregnancy: Multidisciplinary Approach
}

\author{
Jerald Pelayo ${ }^{D},{ }^{1}$ Gabriella Pugliese, ${ }^{2}$ Grace Salacup, ${ }^{1}$ Eduardo Quintero, ${ }^{1}$ Adeeb Khalifeh, ${ }^{3}$ \\ David Jaspan, ${ }^{2}$ and Bhavna Sharma ${ }^{1,4,5}$ \\ ${ }^{1}$ Department of Medicine, Einstein Medical Center Philadelphia, Philadelphia, PA, USA \\ ${ }^{2}$ Department of Obstetrics and Gynecology, Einstein Medical Center Philadelphia, Philadelphia, PA, USA \\ ${ }^{3}$ Division of Maternal Fetal Medicine, Einstein Medical Center Philadelphia, Philadelphia, PA, USA \\ ${ }^{4}$ Division of Pulmonary and Critical Care and Sleep Medicine, Einstein Medical Center Philadelphia, Philadelphia, PA, USA \\ ${ }^{5}$ Sidney Kimmel College of Thomas Jefferson University, Philadelphia, PA, USA
}

Correspondence should be addressed to Jerald Pelayo; pelayoje@einstein.edu

Received 23 July 2020; Revised 13 September 2020; Accepted 27 September 2020; Published 16 October 2020

Academic Editor: Mehmet Doganay

Copyright (C) 2020 Jerald Pelayo et al. This is an open access article distributed under the Creative Commons Attribution License, which permits unrestricted use, distribution, and reproduction in any medium, provided the original work is properly cited.

\begin{abstract}
The rapidly expanding cases of the coronavirus disease 2019 (COVID-19) caused by the severe acute respiratory syndrome coronavirus 2 (SARS-CoV-2) have exposed vulnerable populations, including pregnant women to an unprecedented public health crisis. Recent data show that pregnancy in COVID-19 patients is associated with increased hospitalization, admission of the intensive care unit, and intubation. However, very few resources exist to guide the multidisciplinary team in managing critically ill pregnant women with COVID-19. We report our experience with managing a morbidly obese pregnant woman at 36 weeks' gestation with history of asthma and malignancy who presented with persistent respiratory symptoms at an outside hospital after being tested positive for SARS-CoV-2 polymerase chain reaction (PCR). Early in the course of the hospitalization, patient received remdesivir, convalescent plasma, bronchodilator, systemic steroids, and IV heparin for COVID-19 and concomitant asthma exacerbation and pulmonary embolism. Due to increasing oxygen requirements, she was eventually intubated and transferred to our institution for higher level of care. Respiratory acidosis, severe hypoxemia, and vent asynchrony were managed with vent setting adjustment and paralytics. After 12 hours from spontaneous rupture of her membranes and with stabilization of maternal status, patient underwent a term cesarean delivery for nonreassuring fetal heart tracing. The neonate was discharged on the $2^{\text {nd }}$ day of life, while the patient was extubated on the $6^{\text {th }}$ postpartum day and was discharged to acute inpatient rehabilitation facility on the $19^{\text {th }}$ hospital day. This report highlights the disease progression of COVID-19 in a pregnant woman, the clinical challenges in the critical care aspect of patient management, and the proposed multidisciplinary strategies utilizing an algorithmic approach to optimize maternal and neonatal outcomes.
\end{abstract}

\section{Introduction}

Deemed as one of the most profound public health threats over the past decades, COVID-19 has now been reported from all continents except for Antartica, affecting 7,124,043 individuals in 118 countries with the recorded mortality rate of $5.7 \%$ as of June 8,2010 [1]. It has exposed vulnerable populations, including the pregnant patients, to an unparalleled global health crisis.
Outcomes from multiple studies demonstrated a significant risk of morbidity and mortality among pregnant women infected by influenza, severe acute respiratory syndrome (SARS), and Middle East respiratory syndrome (MERS) compared to nonpregnant women [2-4]. Based on the previous limited data, this particular patient population is not at higher risk for developing severe disease due to COVID-19 compared to the general population $[1,5,6]$. However, the most recent data from the Centers for Disease Control and 


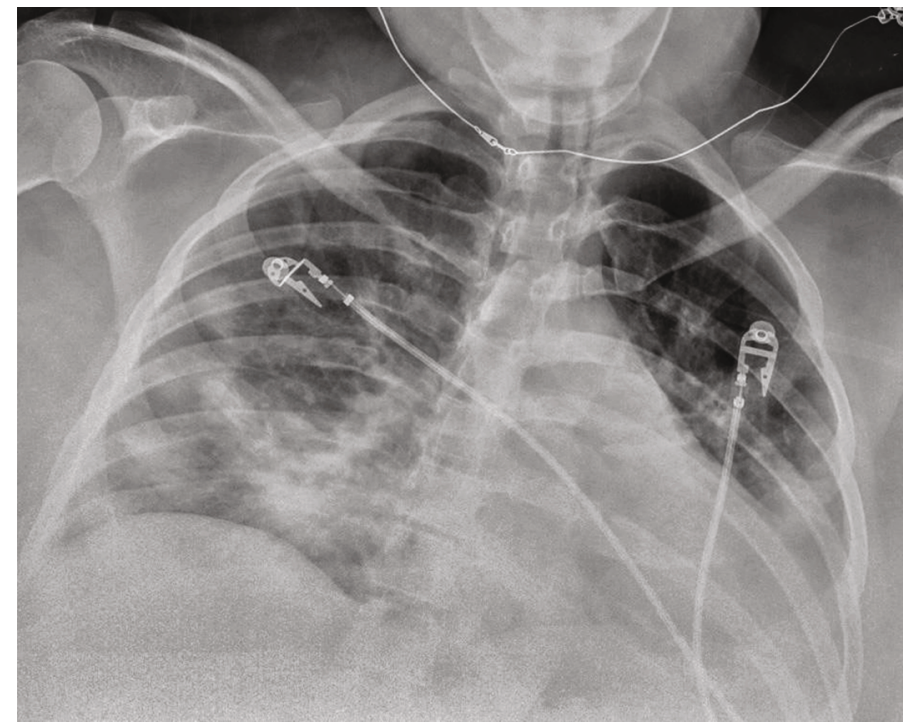

FIGURE 1: Chest radiograph on admission at the outside hospital showing interval worsening of hazy airspace opacities in the bilateral mid to lower lung fields.

Prevention (CDC) showed the pregnant women with COVID-19 are more likely to be hospitalized, admitted to the intensive care unit, and intubated [7].

Data on the effect of COVID-19 in pregnancy in large retrospective study of 119 pregnant women and systematic review of 109 pregnancies showed a $2.7 \%$ to $6.9 \%$ incidence rate of severe infection requiring ICU admission with no maternal deaths $[8,9]$. However, there has been no onesize-fits-all approach in managing critically ill pregnant women with COVID-19 given unique physiologic maternal adaptations and important management and ethical considerations. Furthermore, few contemporary resources are available to guide the multidisciplinary team through decisions regarding intensive care strategies, optimal maternal-fetal surveillance, and route as well as timing of delivery.

We present a case of a woman at 36 weeks' gestation who had a rapid clinical deterioration from COVID-19-related ARDS to highlight clinical challenges and potential strategies through multidisciplinary team approach to optimize maternal and neonatal outcomes.

\section{Case Presentation}

Patient is a 35-year-old G4P1-1-1-2 with a past medical history notable for mild intermittent asthma, hepatitis $\mathrm{C}$, gastric carcinoid tumor, ileal malignant carcinoma S/P hemicolectomy, morbid obesity (body mass index of $51 \mathrm{~kg} / \mathrm{m}^{2}$ ), abdominal hernia s/p mesh repair, bipolar, and anxiety disorder, with a confirmed SARS-CoV2 PCR after a recent known exposure to a positive household contact. She was admitted to a tertiary care hospital after failing self-quarantine measures at home, with persistent nonproductive cough, hemoptysis and worsening shortness of breath without significant response to frequent albuterol nebulization at home. At the time of admission, age of gestation by the last menstrual period (LMP) was $362 / 7$ weeks. At presentation, she was normotensive, tachycardic, and tachypneic but not hypoxic on room air. She had diffuse wheezing and rhonchi on lung auscultation. Significant laboratory findings include thrombocytopenia $(98,000 / \mathrm{uL})$, normocytic anemia with normal haptoglobin level $(145 \mathrm{mg} / \mathrm{ml})$, elevated LDH of $264 \mathrm{IU} / \mathrm{L}$ with mild transaminitis (AST 53 IU/L, ALT 53 IU/L) without schistocytes on peripheral blood smear and normal bilirubin levels, elevated ferritin $(140 \mathrm{ng} / \mathrm{mL})$, and D-dimer $(2810 \mathrm{ng} / \mathrm{mL})$. The nonstress test was reactive with reassuring fetal heart tracing. Chest radiograph (Figure 1) showed worsening bilateral pulmonary opacities compared to the previous (Figure 2). CT pulmonary angiography demonstrated extensive bilateral ground-glass opacities with peripheral distribution, small bilateral pleural effusion, and multiple bilateral intraluminal filling defects of the pulmonary arteries suggestive of pulmonary embolism (Figures 3 and 4).

The patient was admitted to the general medical floor where she received convalescent plasma transfusion, in addition to bronchodilators and intravenous methylprednisolone. She was started on a 10-day course of remdesivir treatment. Over the next four days, she had increasing oxygen requirement from $4 \mathrm{~L}$ to $12 \mathrm{~L}$ via nasal cannula to keep her oxygen saturation above $92 \%$. On the $5^{\text {th }}$ day of admission, patient was noted to be agitated and hypoxic to $88 \%$ on $40 \mathrm{~L}$ of oxygen via a combination of nasal cannula with nonrebreather mask on it. During this time, fetal heart tracing was reassuring. Tocogram showed contractions every 4 to 6 minutes. She was then transferred to the medical intensive care unit (MICU) to attempt high flow nasal cannula (HFNC). After persistent hypoxia on HFNC, the decision was made to intubate the patient. Mechanical ventilation with assist control/volume control settings includes tidal volume (TV) of $500 \mathrm{ml}(7 \mathrm{ml} / \mathrm{kg})$, respiratory rate (RR) of 25 , positive end expiratory pressure (PEEP) of 14 , and fraction of inspired oxygen ( $\mathrm{FiO} 2)$ of $100 \%$. Heparin, cis-atracurium, fentanyl, and propofol drips were initiated and continued. She was then transferred to our institution via air ambulance for higher level of care. 


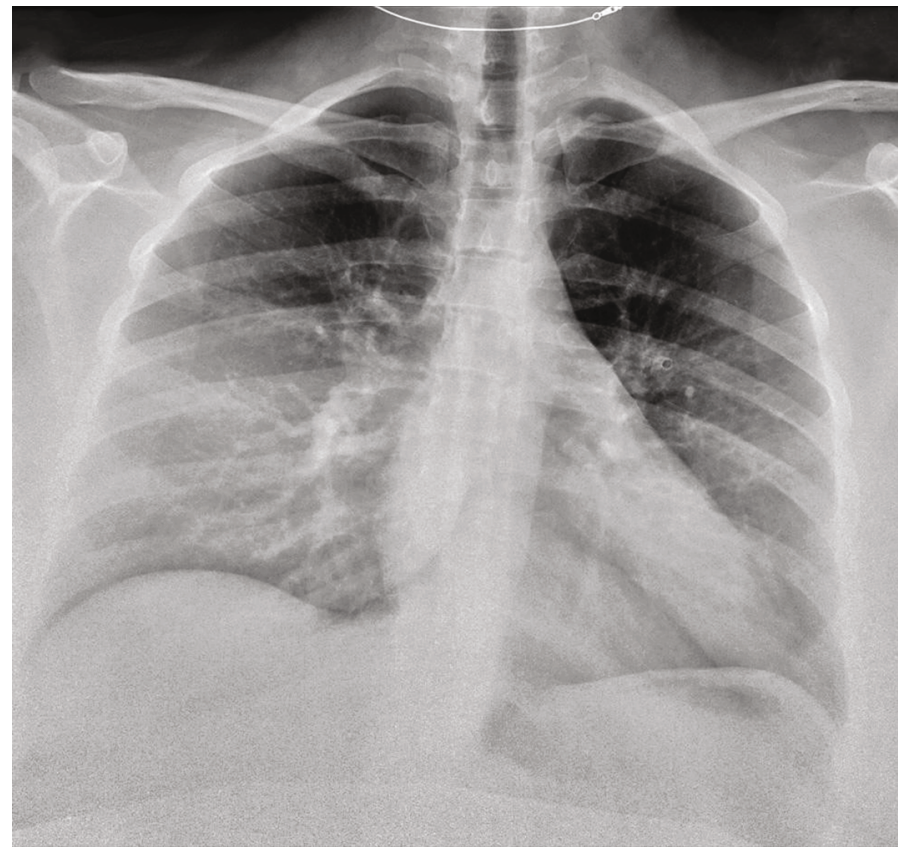

FIGURE 2: Chest radiograph on prior ED visit showing haziness overlying the right mid and lower lung fields.

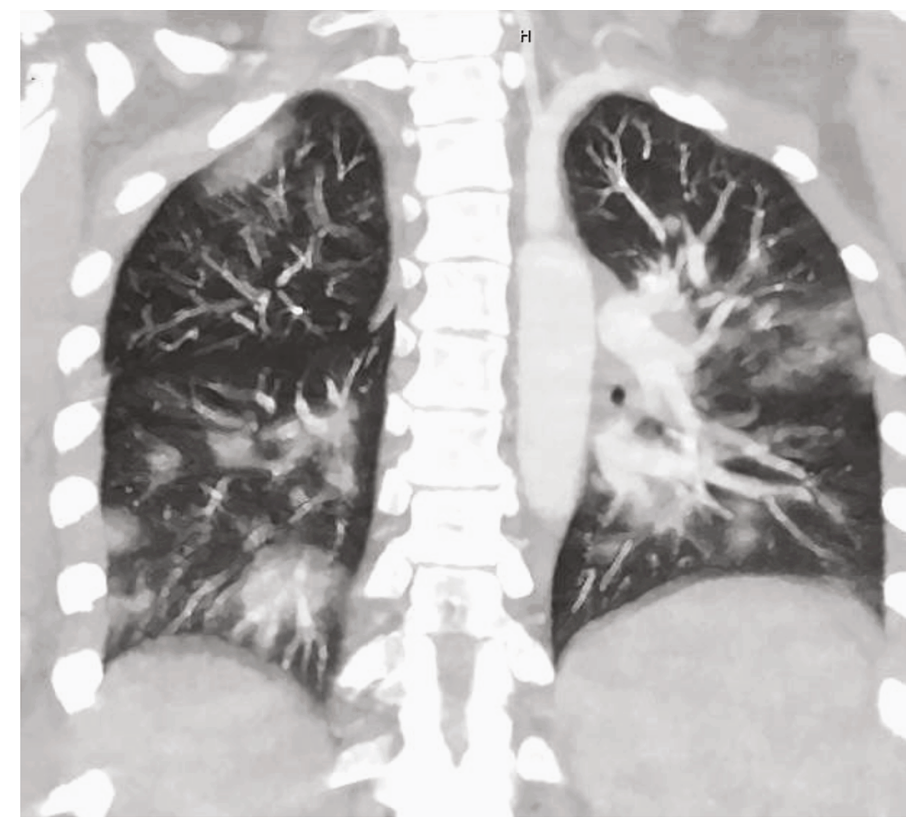

FIGURE 3: CT Pulmonary angiography (coronal section) showing extensive bilateral ground-glass and consolidative opacities with peripheral distribution and small bilateral pleural effusion.

Maternal-fetal medicine (MFM), obstetric, neonatal, and medical intensive care teams were alerted prior to arrival. Patient arrived to our MICU sedated and intubated. She was initially requiring vecuronium pushes to achieve vent synchrony and oxygen saturation above 92\%. Chest radiograph did show worsening of lower lobe predominant diffuse reticular and alveolar airspace opacifications (Figure 5). Mechanical ventilation settings were modified to TV of $460 \mathrm{ml}(6 \mathrm{ml} / \mathrm{kg}), \mathrm{RR}$ of 24 , PEEP of 12 , and $100 \% \mathrm{FiO} 2$. Arterial blood gas $(\mathrm{ABG})$ on this setting includes a $\mathrm{pH}$ of 7.19 ,
$\mathrm{PaCO} 2$ of $63 \mathrm{mmHg}$, and $\mathrm{PaO} 2$ of $80 \mathrm{mmHg}$. The computed $\mathrm{PaO} 2$ : FiO2 ratio was 80 indicative of severe acute respiratory distress syndrome (ARDS). The respiratory rate was increased to $28 /$ minute. On exam, her membranes had spontaneously ruptured, and her cervix was dilated to $4 \mathrm{~cm}$ and $50 \%$ effaced. Bedside ultrasound showed decreased amniotic fluid index and cephalic presentation. The fetal heart rate was 135 beats per minutes (bpm) with overall minimal variability and positive acceleration response on scalp stimulation. Induction of labor with IV oxytocin was started with initial 


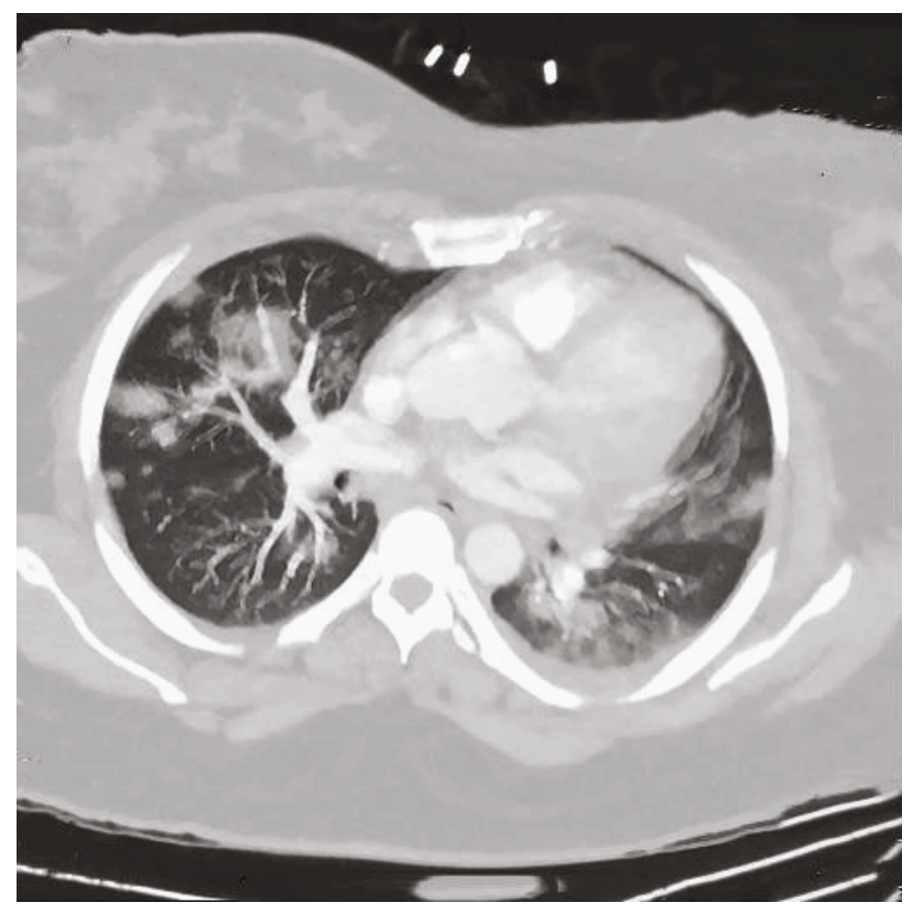

FIGURE 4: CT Pulmonary angiography (axial section) showing extensive bilateral ground-glass and consolidative opacities with peripheral distribution, small bilateral pleural effusion, and multiple bilateral intraluminal filling defects.

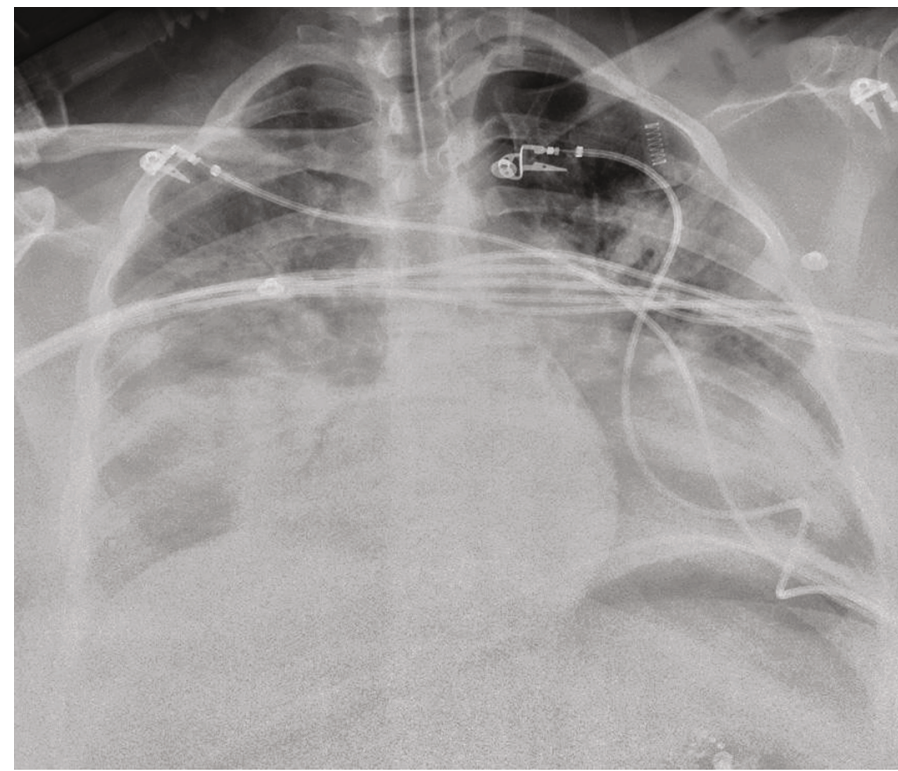

FIGURE 5: Chest radiograph shows interval worsening of bilateral lower lobe predominant diffuse reticular and alveolar airspace opacifications with trace bilateral pleural effusions.

plan to attempt vaginal delivery with the assisted second stage. However, plans for emergency cesarean delivery were in place in the event it was indicated. IV heparin was continued. This plan was reviewed in the multidisciplinary approach including the medical and neonatal intensive care, obstetric, MFM, and anesthesiology teams.

Eight hours after admission, patient was saturating in the high $88-91 \%$ range on a PEEP of 12 and $100 \%$ FiO2. ABG on this setting showed a $\mathrm{pH}$ of $7.21, \mathrm{PaCO} 2$ of $55 \mathrm{mmHg}$, and
$\mathrm{PaO} 2$ of $82 \mathrm{mmHg}$. Plateau pressure was high, ranging between 38 and $40 \mathrm{~cm} \mathrm{H20.This} \mathrm{was} \mathrm{partially} \mathrm{due} \mathrm{to} \mathrm{patient's}$ body habitus, morbid obesity, and a gravid uterus. The severe hypoxemia was deemed potentially harmful to the fetus, and various options were considered to improve patient's oxygenation.

Given her gravid uterus, active labor, and BMI $>50 \mathrm{~kg} / \mathrm{m}^{2}$, prone ventilation was not an option. As a rescue measure, PEEP was then increased to 16 despite the high plateau 
pressure and $\mathrm{RR}$ to $32 /$ minute. She received additional doses of paralytics to maintain vent synchrony. IV heparin was held in preparation for imminent delivery. Given the mother's severe hypoxia and potential for deterioration due to fluid shifts during delivery, extracorporeal membrane oxygenation (ECMO) was also discussed. An emergent discussion was held with another tertiary care hospital within the city with ECMO expertise. The patient was accepted there, in case she required ECMO, and our cardiothoracic surgeon was on board to establish femoral and jugular access in anticipation of emergent ECMO needs in the operating room, with improvement of oxygen status and respiratory acidosis, respectively, as evidenced by $\mathrm{pH}$ of $7.31, \mathrm{PaCO} 243 \mathrm{mmHg}$, and $\mathrm{PaO} 2$ $377 \mathrm{mmHg}$ on repeat ABG. FiO2 was then decreased to $90 \%$.

Cervical dilatation remained relatively unchanged over the next hour or so, with three contractions palpated to be occurring within 10 minutes. Fetal heart monitoring showed minimal variability with no acceleration response on scalp stimulation. In view of this nonreassuring fetal heart tracing, the decision was made to proceed with cesarean delivery in the operating room. The medical intensive care, obstetric, MFM, neonatal intensive care, anesthesiology, cardiothoracic surgery teams were in coordination to ensure the stability of the patient and to address anticipated fetomaternal complications. Patient remained stable throughout the cesarean section and did not require ECMO.

She gave birth to an early term male infant with APGAR scores of 1 at $1^{\text {st }}$ minute, 2 at $5^{\text {th }}$ minute, and 4 at $10^{\text {th }}$ until the $20^{\text {th }}$ minute. As anticipated prior to delivery, the neonate required invasive mechanical ventilation, due to prolonged exposure to sedatives and paralytics from the mother. He needed bag mask ventilation with $100 \% \mathrm{FiO} 2$ without return of spontaneous breathing. The heart rate was less than 60 beats per minute (bpm) requiring 30-second chest compression. After 5 minutes, the heart rate was $120 \mathrm{bpm}$; however, he was hypoxic to $85 \%$ while on $100 \% \mathrm{FiO} 2$ necessitating mechanical ventilation. Neonate was then admitted to neonatal ICU which was tested for SARS-CoV2 which turned out negative twice. The following day, patient's baby was extubated to room air and was subsequently released on his $3^{\text {rd }}$ day of life to his aunt for primary care as his father was still positive for SARS-COV2.

In the interim, patient received a five-day course vancomycin and ceftriaxone for sputum culture growing $\mathrm{Hae}$ mophilus influenzae and methicillin-resistant Staphyloccus aureus. There was a very low suspicion for bacterial superinfection given transient fever, mild leukocytosis with lymphocytic predominance, and absence of consolidation on chest radiograph. Most of the treatment were geared towards supportive management for COVID-19 and acute respiratory distress syndrome.

On the other hand, patient remained intubated, with decreasing oxygen requirements in the postpartum period. Her hypoxia improved significantly after delivery, despite a newly diagnosed cardiomyopathy as evidenced with $45 \%$ ejection fraction on echocardiography. IV heparin was resumed 6 hours postpartum. She was eventually extubated to bilevel positive airway pressure (BiPAP) on the $6^{\text {th }}$ day of intubation. She developed postextubation stridor necessitat- ing administration of racemic epinephrine and solumedrol. She finished the 10-day course of remdesivir and 5-day course of IV methylprednisolone. IV heparin was also transitioned to subcutaneous enoxaparin. Patient was discharged to acute inpatient rehabilitation facility after 19 days of hospitalization.

\section{Discussion}

This case highlights the clinical course of the COVID-19 infection in a pregnant woman with multiple comorbidities, the importance of taking into consideration physiologic maternal adaptations, and the intricate critical care aspects in managing the patient with anticipated fetomaternal complications from COVID-19-related ARDS and as well as the multidisciplinary approach to optimize maternal and neonatal outcomes (Figure 6).

Patient's current pregnancy with her comorbidities including malignancy, asthma , and morbid obesity further complicated by pulmonary embolism during her hospitalization may have largely contributed to the development of severe acute respiratory distress syndrome (ARDS) in the setting of COVID-19 pneumonia [7-11].

Many potential challenges confronted the medical and neonatal intensive care, obstetric, and maternal fetal medicine teams in managing patient's severe hypoxemia with severe ARDS. It is of paramount importance to take into consideration maternal physiologic adaptations to pregnancy. Apart from leaving the woman more vulnerable to cell-mediated viral infection such as COVID-19, pregnant women are more susceptible to rapid cardio-pulmonary decompensation due to reduced cardiac and pulmonary reserves [12]. Particularly in the third trimester, the gravid uterus decreases functional residual capacity and expiratory reserve volume, which can potentially increase the risk of severe hypoxemia especially those who are critically ill [13]. Moreover, the physiologic adaptations to labor, delivery, and the immediate postpartum should also be considered as these could exacerbate the dysregulated inflammatory cascade in the setting of an underlying severe systemic infection. These physiologic changes include significant fluid shifts between the interstitial, intracellular, and intravascular compartments, maximization of the maternal cardiac output, autotransfusion of up to $500 \mathrm{~mL}$ of blood back into the intravascular compartment, catecholamine surge, and release of inflammatory mediators within the endothelium [11]. These in the setting of COVID-19 infection could place the patient at a higher risk for developing endothelial dysfunction, pulmonary edema, myocardial edema, and cardiac dysfunction [14]. Such considerations were at the forefront, in the minds of the entire medical and surgical team in optimizing patient's oxygenation while she was mechanically ventilated before, during and after the delivery.

While patient's labor was induced with IV oxytocin, options to improve patient's oxygenation prior to the contemplated delivery were maximized. First, ventilatory goals include consideration for the diminished functional residual capacity, higher PEEP requirement, greater physiologic tidal volume, and less lung compliance from higher innate plateau 


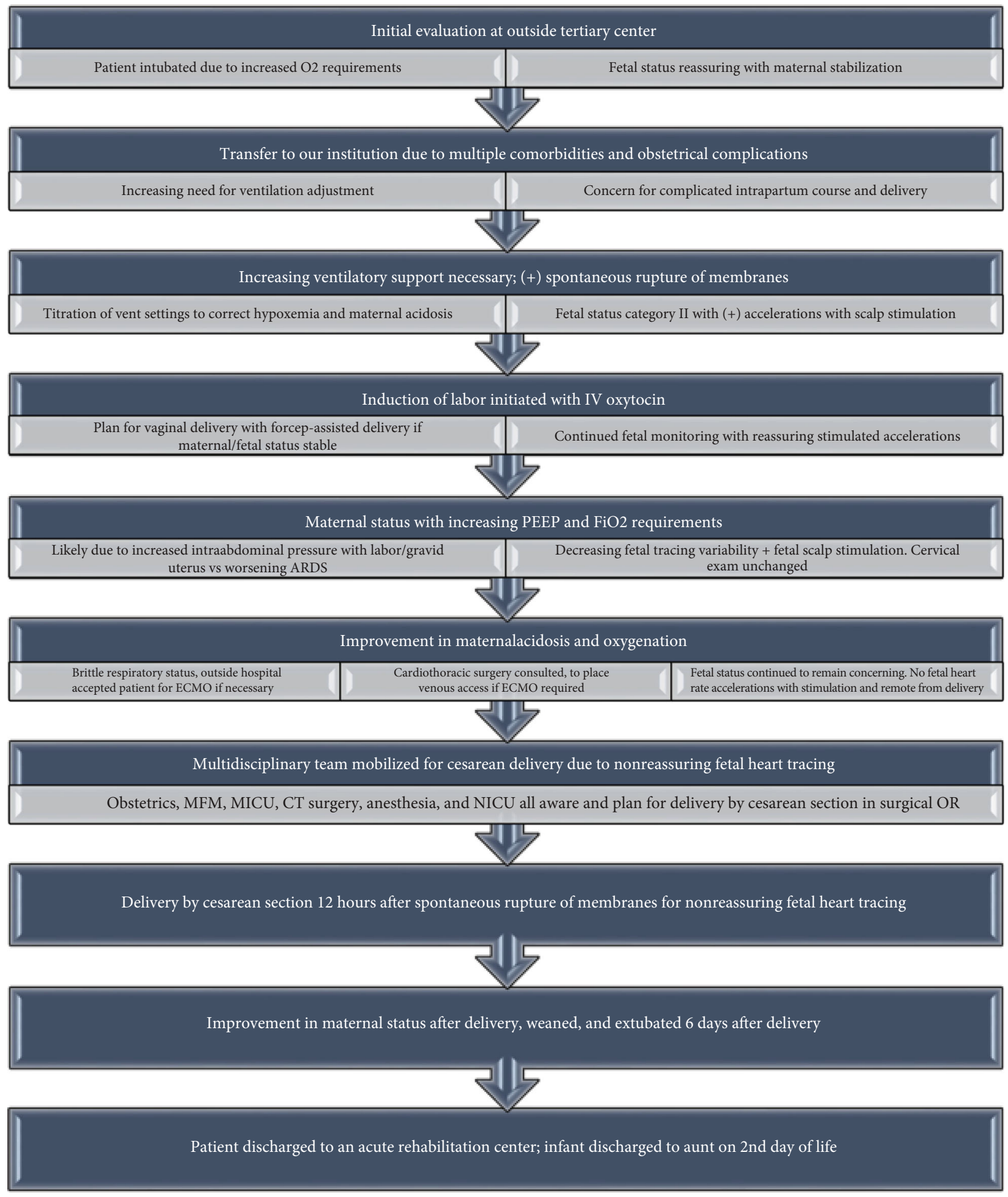

FIGURE 6: Summary of multidisciplinary management and outcomes of our COVID-19 pregnant patient.

pressures due to diaphragmatic compression by the gravid uterus and rotund abdomen and chest wall compression from enlarged breast tissue $[12,15]$. These presented a challenge to the "lung protective" strategy for mechanical ventilation in pregnant patients. It was then reasonable to increase the PEEP and $\mathrm{FiO} 2$ of this patient to meet oxygenation target.
Second is the use of neuromuscular blocking agents with sedation in the early phase of ARDS. It was found to have added clinical benefit by minimizing the manifestations of ventilator-induced lung injury by reducing pulmonary and systemic production of inflammatory mediators [14]. Patient did receive cis-atracurium drip in another hospital before 


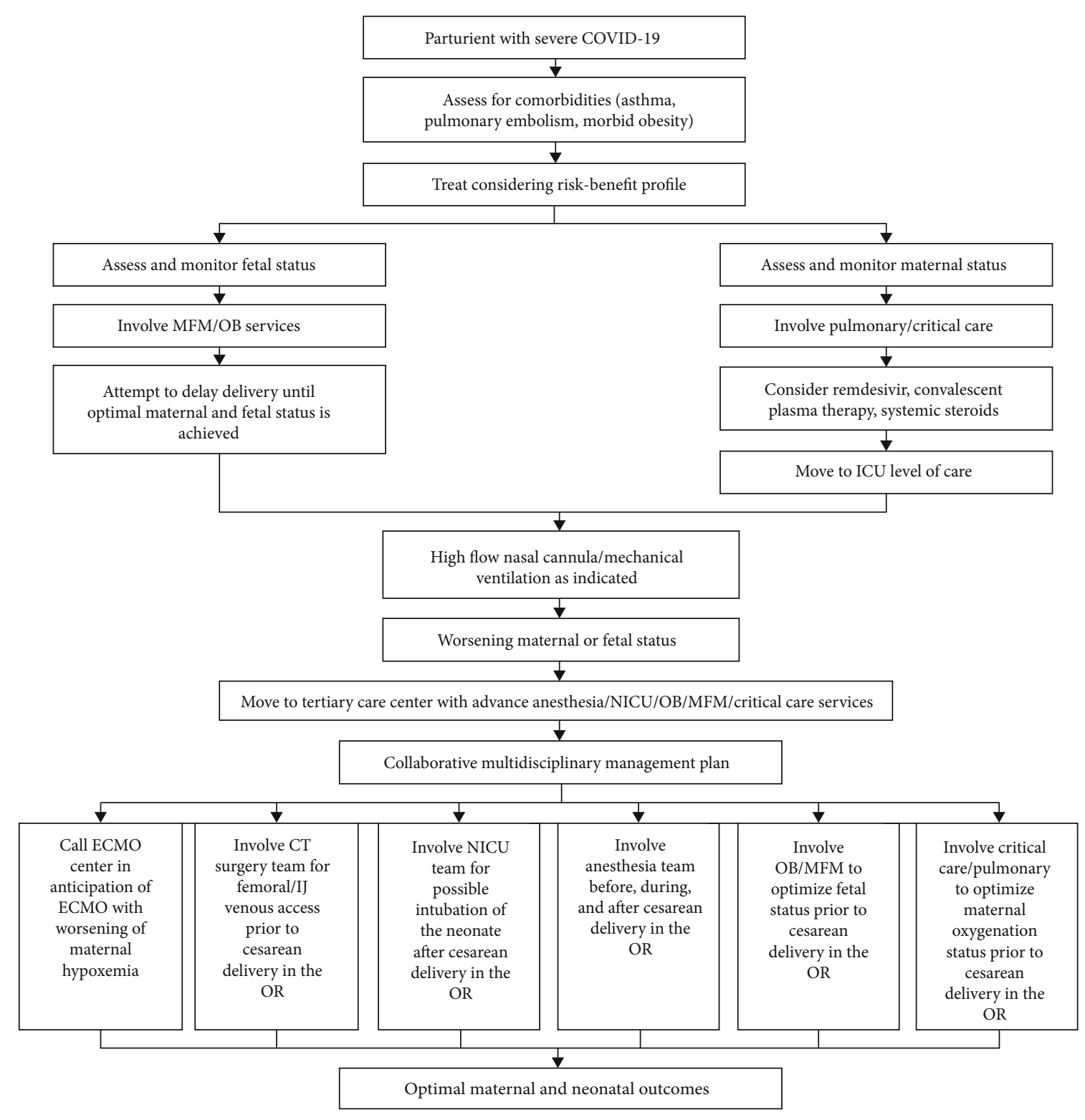

FIGURE 7: Proposed algorithm for multidisciplinary management of a parturient with severe COVID-19.

transfer and intermittent vecuronium pushes while admitted to our ICU. Patient ended up with cesarean delivery at term for nonreassuring fetal heart status with stable maternal status after correcting respiratory acidosis and achieving oxygenation goals. This is congruous with the result of a systematic review and meta-analysis showing increased cesarean delivery rate among pregnant women with COVID-19 [16].

Although the safety and efficacy of prone ventilation in pregnancy has been documented $[17,18]$, patient's weight, size and active labor precluded the performance of this maneuver. Pregnancy is also a contraindication for prone ventilation per our institutional policy. Thus, venovenous ECMO was considered as a life-saving salvage therapy in the event of worsening oxygenation and hemodynamic compromise during and after the delivery [19]. Cardiothoracic surgeon placed central venous access in the internal jugular and femoral veins prior to the cesarean delivery, in anticipa- tion of ECMO. In the interim, patient was already accepted in another tertiary hospital for ECMO should the need arise.

As most of the trials for treatment of COVID-19 exclude pregnant women, few agents although available remain investigational in terms of their clinical utility. These include remdesivir, systemic steroids, and convalescent plasma which are offered in different hospitals under compassionate-use protocol [20-22]. After a lengthy discussion, patient and her husband provided consent for the administration of the remdesivir and convalescent plasma early in the course of patient's hospitalization. Timely initiation of bronchodilator and steroid therapy, as well as IV heparin for asthma exacerbation and pulmonary embolism, respectively, might have afforded additional benefit leading to patient's excellent overall clinical outcomes.

As to the neonatal outcome, patient's baby improved clinically after resuscitation and mechanical ventilation for 
24 hours. Cardiorespiratory depression was likely secondary to prolonged maternal exposure to sedation and paralytics. The SARS-CoV-2 PCR test of the baby turned out negative which is consistent with recent findings documenting no evidence of vertical transmission in women with COVID-19 pneumonia $[6,23]$.

A proposed algorithmic approach to the management of a parturient with severe COVID-19 is included in this report (Figure 7). This entailed valuable input from multiple disciplines involving the medical and neonatal intensive care, obstetric, MFM, anesthesiology, and cardiothoracic surgery teams. A framework utilizing direct open communication that optimized team dynamics was established, along with active involvement of patient's husband in the decisionmaking vis-à-vis available therapeutic options. Maternal and neonatal outcomes were excellent as a result of highly coordinated care through a multidisciplinary approach.

\section{Data Availability}

The data used to support the findings of this study are included within the article.

\section{Conflicts of Interest}

None of the authors have any conflicts of interest to disclose.

\section{References}

[1] World health organization, "COVID19 situation reports," 2020, https://www.who.int/emergencies/diseases/novelcoronavirus-2019/situation-reports.

[2] S. A. Rasmussen, D. J. Jamieson, and T. M. Uyeki, "Effects of influenza on pregnant women and infants. Am J 405," Obstetrics and Gynecology, vol. 207, Supplement 3, pp. S3-S8, 2012.

[3] M. Silasi, I. Cardenas, K. Racicot, J.-Y. Kwon, P. Aldo, and G. Mor, "Viral infections during pregnancy," American Journal of Reproductive Immunology, vol. 73, no. 3, pp. 199-213, 2015.

[4] D. A. Schwartz, "An analysis of 38 pregnant women with COVID-19, their newborn infants, and maternal-fetal transmission of SARS-CoV-2: maternal coronavirus infections and pregnancy outcomes," Archives of Pathology \& Laboratory Medicine, vol. 17, 2020.

[5] Centers for disease control and prevention, "COVID19. People who need to take extra precautions," https://www.cdc.gov/ coronavirus/2019-ncov.

[6] H. Chen, J. Guo, C. Wang et al., "Clinical characteristics and intrauterine vertical transmission potential of COVID-19 infection in nine pregnant women: a retrospective review of medical records," The Lancet, vol. 395, no. 10226, pp. 809$815,2020$.

[7] S. Ellington, P. Strid, V. T. Tong et al., "Characteristics of women of reproductive age with laboratory-confirmed SARS-COV-2 infection by pregnancy status - united States, January 22-June 7, 2020," MMWR. Morbidity and Mortality Weekly Report, vol. 69, no. 25, pp. 769-775, 2020.

[8] J. Yan, J. Guo, C. Fan et al., "Coronavirus disease 2019 in pregnant women: a report based on 116 cases," American Journal of Obstetrics and Gynecology, vol. 223, no. 1, pp. 111.e1111.e14, 2020
[9] M. Zaigham and O. Andersson, "Maternal and perinatal outcomes with COVID-19: a systematic review of 108 pregnancies," Acta Obstetricia et Gynecologica Scandinavica, vol. 99, no. 7, pp. 823-829, 2020.

[10] Centers for disease control and prevention, "COVID 19. People with certain medical conditions," https://www.cdc.gov/ coronavirus/2019-ncov/need-extra-precautions/people-withmedical-conditions.html\#cancer.

[11] L. Xu, M. Yaqian, and G. Chen, "Risk factors for severe corona virus disease 2019 (COVID-19) patients : a systematic review and meta analysis," 2020.

[12] W. Schettler, Y. Al Ahwel, and A. Suhag, "Severe ARDS in COVID-19-infected pregnancy: obstetric and intensive care considerations," American Journal of Obstetrics \& Gynecology $M F M$, vol. 2, 2020.

[13] C. M. Oxford and J. Ludmir, "Trauma in pregnancy," Clinical Obstetrics and Gynecology, vol. 52, no. 4, pp. 611-629, 2009.

[14] A. Juusela, M. Nazir, and M. Gimovsky, "Two Cases of COVID-19 Related Cardiomyopathy in Pregnancy," American Journal of Obstetrics \& Gynecology MFM, vol. 2, 2020.

[15] M. D. Eisner, T. Thompson, L. D. Hudson et al., "Efficacy of low tidal volume ventilation in patients with different clinical risk factors for acute lung injury and the acute respiratory distress syndrome," American Journal of Respiratory and Critical Care Medicine, vol. 164, no. 2, pp. 231-236, 2001.

[16] J. Bourenne, S. Hraiech, A. Roch, M. Gainnier, L. Papazian, and J. M. Forel, "Sedation and neuromuscular blocking agents in acute respiratory distress syndrome," Annals of Translational Medicine, vol. 5, no. 14, p. 291, 2017.

[17] A. T. Dennis, L. Hardy, and L. Leeton, "The prone position in healthy pregnant women and in women with preeclampsia - a pilot study," BMC Pregancy Childbirth, vol. 18, no. 1, p. 445, 2018.

[18] M. Akatsuka, H. Tatsumi, N. Yama, and Y. Masuda, "Therapeutic evaluation of computed tomography findings for efficacy of prone ventilation in acute respiratory distress syndrome patients with abdominal surgery," The Journal of Critical Care Medicine, vol. 6, no. 1, pp. 32-40, 2020.

[19] C. M. Webster, K. A. Smith, and T. A. Manuck, "Extracorporeal membrane oxygenation in pregnant and postpartum women: a ten-year case series," American Journal of Obstetrics \& Gynecology MFM, vol. 2, no. 2, p. 100108, 2020.

[20] M. Wang and R. Cao, "Remdesivir and chloroquine effectively inhibit the recently emerged novel coronavirus (2019-nCoV) in vitro," Cell Research, vol. 30, no. 3, pp. 269-271, 2020.

[21] H. Li, Y. M. Wang, J. Y. Xy, and B. Cao, "Potential antiviral therapeutics for 2019 coronavirus," Zhonghua Ji He He Hu Xi Za Zhi, vol. 43, no. 3, pp. 170-172, 2020.

[22] V. Bhergella, "Coronavirus disease 2019 (COVID-19): pregnancy issues," https://www.uptodate.com/contents/ coronavirus-disease-2019-covid-19-pregnancy-issues.

[23] D. Di Mascio, A. Khalil, G. Saccone et al., "Outcome of coronavirus spectrum infections (SARS, MERS, COVID-19) during pregnancy: a systematic review and meta-analysis," American Journal Of Obstetrics \& Gynecology MFM, vol. 2, no. 2, p. 100107, 2020. 